\title{
Challenge of Using Antiplatelet Drugs in Patients with Primary Immune Thrombocytopenia and Recently Implanted Coronary Stents-Splenectomy as a Therapeutic Option
}

\author{
${ }^{1}$ Hematology Department, KBC Bezanijska kosa, Belgrade, Serbia \\ ${ }^{2}$ Cardiology Department, KBC Bezanijska kosa, Belgrade, Serbia \\ ${ }^{3}$ Clinic for Hematology, Clinical Center of Serbia, Belgrade, Serbia \\ ${ }^{4}$ Medical School, University of Belgrade, Serbia
}

Dragomir Marisavljevic ${ }^{1,4^{*}}$, Olivera Markovic ${ }^{1,4}$, Marija Zdravković ${ }^{2,4}$, Saša Hinić ${ }^{2}$, Nada Suvajdžić-Vuković ${ }^{3,4}$ and Branka Filipović $^{1,4}$

"Corresponding author: Dragomir Marisavljevic, Hematology Department, KBC "Bezanijska kosa", Belgrade, Serbia, Tel: +381-65-3357-090; E-mail: maris@tehnicom.net

Received date: Oct 28, 2017; Accepted date: Nov 10, 2017; Published date: Nov 18, 2017

Copyright: ( 2017 Dragomir Marisavljevic, et al. This is an open-access article distributed under the terms of the Creative Commons Attribution License, which permits unrestricted use, distribution and reproduction in any medium, provided the original author and source are credited.

\begin{abstract}
Background: Immune thrombocytopenic purpura (ITP) in the patients with implanted coronary stents is related with serious risks of haemorrhage related to dual antiplatelet therapy on the one hand and stent thrombosis if antiplatelet therapy is interrupted on the other hand. Therefore, the main objective in these patients is the correction of thrombocytopenia and continuous use of antiplatelet drugs.

Case report: We present the patient with implanted stents after acute myocardial infarction (AMI) and severe ITP who was successfully treated with splenectomy. After the patient experienced AMI, primary percutaneous coronary intervention $(\mathrm{PCl})$ with implantation of stents has been performed. Thrombocytopenia $\left(21 \times 10^{9} / \mathrm{L}\right)$ was registered for the first time after $\mathrm{PCl}$ intervention when it has been noticed a massive hematoma of whole right arm at the site of radial artery puncture. Immediately after the intervention dual antiplatelet therapy and prednisone has been started. Since corticosteroids and azathioprine treatment proved unsuccessful (platelet count $<10 \times 10^{9} / \mathrm{L}$ ), the patient has been prepared for splenectomy with intravenous immunoglobulins. As the platelet count was in stable range (40-50 $\times 10^{9} / \mathrm{L}$ ) after splenectomy, antiplatelet therapy has been readministered safely.
\end{abstract}

Conclusion: As there are no definitive guidelines for treatment of patients with ITP and implanted stents, the treatment should be individualized to minimize risk of hemorrhagic as well as thrombotic complications. Our case suggests that splenectomy is an available and safe treatment for these patients. However, decision on splenectomy is a challenge; and before the surgical intervention the risk-benefit assessment should be considered.

Keywords: Immune thrombocytopenia; STEMI; Percutaneus coronary intervention; Antiplatelet drugs

\section{Introduction}

Acute myocardial infarction (AMI) is very infrequent in patients with primary immune thrombocytopenia (ITP) and poses serious management problem in which a good balance between prevention of thrombosis and haemorrhagic risk must be achieved [1-4]. The main objectives in these patients are correction of thrombocytopenia and continuous administration of antiplatelet drugs. There are only a few reports of AMI in ITP patients treated by primary percutaneous coronary intervention (PCI) and dual antiplatelet therapy. Pretreatment with some of the modalities (corticosteroids, intravenous immunoglobulins (IVIg), agonists of thrombopoietin receptors, danazol and platelet transfusions) was required in a number of those patients [1-4].

\section{Case Report}

We present here the case of a 43 -year-old male with simultaneous occurrence of ITP and AIM in whom the splenectomy was successfully performed after the failure of standard medicamentous therapy. In February 2016, after AIM was diagnosed, primary PCI with implantation of two stents in Left Descending Coronary (LAD) artery was performed (drug-eluting stent-DES in mid segment of LAD, Xience $2.75 \times 28 \mathrm{~mm}$; bare-metal stent in the proximal segment of LAD, Integrity RX $3.5 \times 18 \mathrm{~mm})$. Thrombocytopenia $\left(21 \times 10^{9} / \mathrm{L}\right)$ in our patient, confirmed by a peripheral blood smear, was registered for the first time after coronary angiography when the massive hematoma at the site of the radial artery punctures arises. After a detailed haematological work-up, a diagnosis of ITP was established. Immediately after PCI dual antiplatelet therapy and prednisone (40 $\mathrm{mg}$ ) have been initiated. Blood counts performed throughout the hospitalization showed a gradual increase of platelet count up to $70 \times$ $10^{9} / \mathrm{L}$. The patient was discharged with dual antiplatelet therapy (acetylsalicylic acid-ASA and clopidrogel) and prednisone $(20 \mathrm{mg})$. Three weeks after the AMI the patient was admitted to the Hematology department due to thrombocytopenia $\left(21 \times 10^{9} / \mathrm{L}\right)$ and skin bleeding. Dual antiplatelet therapy was withheld. Prolonged treatment with corticosteroids and azathioprine proved unsuccessful (platelet count $<10 \times 10^{9} / \mathrm{L}$, presence of haemorrhagic syndrome) (Figure 1) . 
Citation: Marisavljevic D, Markovic O, Zdravkovic M, Hinic S, Suvajdžic-Vukovic N, et al. (2017) Challenge of Using Antiplatelet Drugs in Patients with Primary Immune Thrombocytopenia and Recently Implanted Coronary Stents-Splenectomy as a Therapeutic Option. Adv Pharmacoepidemiol Drug Saf 6: 220. doi:10.4172/2167-1052.1000220

Page 2 of 3

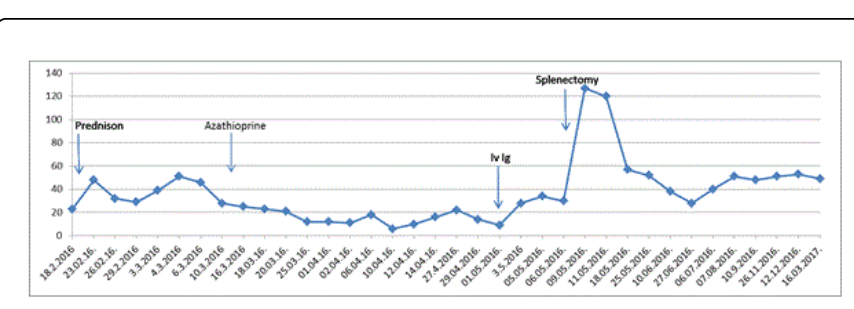

Figure 1: Changes of platelets number in relation to applied therapies.

As thrombocytokinetic examination using in ${ }^{111}$ showed shortened platelet life span ( $24 \mathrm{~h}$, reference range 7 to 10 days) and predominant sequestration of platelets in the spleen, the patient was prepared for splenectomy with IVIg. After splenectomy the patient achieved a stabile partial remission (platelet count $40-50 \times 10^{9} / \mathrm{L}$ ) and dual antiplatelet therapy has been readministered safely. At the last followup (June, 2017) a mild thrombocytopenia $\left(53 \times 10^{9} / \mathrm{L}\right)$ was registered without clinical signs of bleeding and antiplatelet therapy (ASA, 100 mg) was continued.

\section{Discussion}

Acute coronary events occurs in patients with ITP regardless of their platelet count, which could range from normal to as low as $2 \times$ $10^{9} / \mathrm{L}[1,3-5]$. The precise mechanism of arterial thrombosis in ITP is unknown, but several explanations have been offered: Presence of large, immature, prothrombotic platelets, elevated platelet microparticles which promote coagulation, presence of antiphospholipid antibodies and anti-IIb/IIIa antibodies which target both platelets and endothelial cells due to antigenic mimicry [6].

Treatment of ITP in the patients with implanted coronary stents is a clinical conundrum, because there is no guidance or official recommendation for treatment of such patients. Besides, a number of treatment modalities for ITP increase susceptibility to thrombotic events due to either abrupt rise of the platelet count with emergence of immature prothrombotic platelets (rituximab, IVIg) or increased plasma viscosity (IVIg, platelet transfusion) or metabolic changes which promote atherosclerosis (steroids) [2]. The thrombopoietin receptor agonists (TPO-RA) and danazol are also related to thrombotic complications [7].

It seems that antiplatelet therapy is safe and well tolerated chronically when platelet count is $>30 \times 10^{9} / \mathrm{L}$ and should be discontinued only during the bleeding episode [6]. Withdrawal of clopidogrel and maintenance of antiplatelet therapy with aspirin alone has been described, too [1]. However, in view of the persistence of severe thrombocytopenia accompanied by haemorrhagic syndrome after the administration of conventional therapy we decided to perform splenectomy in our patient.

Some authors emphasize that special attention should be paid to the type of the stent in ITP patients, as it will influence the duration of dual antiplatelet therapy $[1,8]$. Namely, some experts recommend albuminal biodegradable polymer DES of new generation since dual antiplatelet therapy with acetylsalicylic acid and clopidogrel could be administered for a shorter period of time to allow stent endothelialisation [9].
The patients with ITP and coronary stents require a tailored medical and interventional management. The case we reported suggests that splenectomy is an available and safe treatment for ITP patients with recently implanted coronary stents, providing stabile platelet count. Decision of splenectomy is a challenge. Namely, splenectomy, a treatment often reserved for refractory ITP, can predispose to venous thromboembolism. Before the surgery, the risk-benefit assessment should be performed.

In the case of ITP reccurence after splenectomy, readministration of corticosteroids might be considered. In emergency situations, injection of IVIg may be appropriate. For a patient with symptomatic corticosteroid-dependent ITP the use of azatioprine, mycophenolate mofetil or vinca alcaloides could be appraised. Concerning TPO-RA, they are discouraged in patients with high thrombotic risk. However, a case of eltrombopag use in a patient with recent acute coronary syndrome has been published recently [1]. As there are no definitive guidelines, treatment should be individualized to minimize the risk of haemorrhagic as well as thrombotic complications.

\section{Conclusion}

We report the unique case of a patient with simultaneous occurrence of AIM and ITP. Thrombocytopenia was not registered before performing PCI with implantation of two stents to his LAD. After the initial good treatment response to corticosteroids which allowed the continuation of dual antiplatelet therapy the patient showed subsequently corticoresistance and unresponsiveness to azathioprine. Considering his "favourable" thrombocytokinetic profile (platelet premature sequestration predominantly in the spleen) splenectomy appeared a reasonable treatment approach. He was prepared with IVIg for surgery. After splenectomy, patient achieved a stabile partial remission allowing continuation of antiplatelet therapy. The patient is at high risk for in-stent restenosis and treating his thrombocytopenia may worsen thrombosis. Treatment for such patients needs to be individualized and more research is needed in this subject to make guidelines to therapy.

\section{Conflict of Interest}

The authors have no conflicts of interest.

\section{References}

1. Bermejo N, Siguenza R, Ibanez F (2017) Management of primary immune thrombocytopenia with Eltrombopag in a patient with recent acute coronary syndrome. Rev Esp Cardiol 70: 56-57.

2. Paolini R, Fabris F, Cella G (2000) Acute myocardial infarction during treatment with intravenous immunoglobulin for idiopathic thrombocytopenic purpura. Am J Hematol 65:177-178.

3. Fong MC, Chen KC, Leu HB, Chen LC (2006) Coronary revascularization in a patient with immune thrombocytopenic purpura. J Chin Med Assoc 69: 436-438.

4. Neskovic AN, Stankovic I, Milicevic P, Aleksic A, Vlahovic-Stipac A, et al. (2010) Primary PCI for acute myocardial infarction in a patient with idiopathic thrombocytopenic purpura. A case report and review of the literature. Herz 35: 43-49.

5. Kim JH, Park KU, Chun J, Kim SH, Nah DY (2006) Primary percutaneous coronary intervention for acute myocardial infarction with idiopathic thrombocytopenic purpura: A case report. J Korean Med Sci 21:355-357.

6. Torbey E, Yacoub H, McCord D, Lafferty J (2013) Two cases and review of the literature: Primary percutaneous angiography and antiplatelet 
Citation: Marisavljevic D, Markovic O, Zdravkovic M, Hinic S, Suvajdžic-Vukovic N, et al. (2017) Challenge of Using Antiplatelet Drugs in Patients with Primary Immune Thrombocytopenia and Recently Implanted Coronary Stents-Splenectomy as a Therapeutic Option. Adv Pharmacoepidemiol Drug Saf 6: 220. doi:10.4172/2167-1052.1000220

Page 3 of 3

management in patients with immune thrombocytopenic purpura. ISRN Hematol 2013: 1-4.

7. Rayoo R, Sharma N, Van Gaal WJ (2012) A case of acute stent thrombosis during treatment with the thrombopoietin receptor agonist peptideRomiplostim. Heart Lung Circ 21: 1820-1824.

8. Stouffer GA, Hirmerova J, Moll S, Rubery B, Napoli M, et al. (2004) Percutaneous coronary intervention in a patient with immune thrombocytopenia purpura. Catheter Cardiovasc Interv 61: 364-367.
9. Urban P, Meredith IT, Abizaid A, Pocock SJ, Carrié D, et al. (2015) Polymer-free drug-coated coronary stents in patients at high bleeding risk. N Engl J Med 373: 2038-2047. 